Relations industrielles

Industrial Relations

\title{
Les congés annuels payés dans le Québec
}

\section{Charles Bélanger}

Volume 3, numéro 10, juin 1948

URI : https://id.erudit.org/iderudit/1023636ar

DOI : https://doi.org/10.7202/1023636ar

Aller au sommaire du numéro

Éditeur(s)

Département des relations industrielles de l’Université Laval

ISSN

0034-379X (imprimé)

1703-8138 (numérique)

Découvrir la revue

Citer cet article

Bélanger, C. (1948). Les congés annuels payés dans le Québec. Relations industrielles / Industrial Relations, 3(10), 148-151.

https://doi.org/10.7202/1023636ar

Tous droits réservés @ C Département des relations industrielles de l’Université Laval, 1948
Ce document est protégé par la loi sur le droit d'auteur. L’utilisation des services d'Érudit (y compris la reproduction) est assujettie à sa politique d'utilisation que vous pouvez consulter en ligne.

https://apropos.erudit.org/fr/usagers/politique-dutilisation/ 


\section{LES CONGÉS ANNUELS PAYÉS DANS LE QUÉBEC}

\section{Charles BÉLANGER}

L'établissement d'un régime légal de congés annuels payés dans la province de Québec, devenu possible à la suite du pouvoir accordé à cet effet par la Législature à la Commission du salaire miminimum, par un amendement à sa loi organique ${ }^{1}$, n'apportait pas une solution à un problème aussi vital que le salaire, par exemple, mais il marquait une étape importante dans l'amélioration des conditions sociales des travailleurs. Ce régime, il faut le remarquer, n'était pas entièrement nouveau puisque déjà un grand nombre de salariés jouissaient de congés payés, annuels et autres.

Nous tenterons dans cet article de recunstituer brièvement les principales étapes qui ont facilité l'avènement d'une réglementation d'ordre public en ce domaine: l'ordonnance no 3 de la C $\mathrm{sm}$ mission du salaire minimum. Nous nous attarderons ensuite à considérer les principales modalités d'application de cette ordonnance.

Des heures de loisirs isolées, des jours fériés chômés ${ }^{2}$, procuraient aux travailleurs certains avantages physiques et moraux. Ces solutions partielles étaient insuffisantes: un arrêt prolongé du travail devenait nécessaire.

Avant l'intervention récente de l'Etat dans les relations du travail, quelques employeurs accordaient des congés prolongés avec paye surtout dans la grande entreprise. L'habitude datait déjà depuis longtemps dans les services administratifs. Sauf quelques rares exceptions, les humbles salariés n'y avaient aucun droit puisque les employeurs n'étaient pas tenus légalement d'en accorder.

La montée de l'associationnisme, surtout chez les ouvriers, et la négociation des conventions collectives devenue institution, ont favorisé le développement de cette mesure. Mais encore, de façon générale, seuls ceux pour qui les syndicats réclamaient $y$ avaient droit. Si l'on considère que jusqu'à récemment, à peine dix pour cent des salariés étaient groupés en syndicats, on peut se sendre compte, même si l'on y ajoute les emplnyés

(1) Loi du salaire minimum, S.R.Q., 1941, c. 164 et amendements.

(2) On trouvera à la suite de cet article deux tableaux analytiques des conventions collectives de travail prévoyant des jours fériés chômés payés et non payés. des services publics et ceux des grandes entreprises dont la politique sociale avait suffisamment évolué, que les congés payés n’étaient le partage que de certains privilégiés. La grande majorité des travailleurs ne pouvaient aucunement en bénéficier sans en faire eux-mêmes les frais.

L'opinion publique, durant les années de guerre, a été graduellement préparée à cette éventualité. On se souvient, en effet, que les décrets de stabilisation des salaires ont empêché commie mesure anti-inflationnaire la majorité des travailleurs d'obtenir une amélioration de leurs c.mditions de rémunération. Cependant, le gouvernement fédéral a adopté une politique sur les congés annuels payés et le Conseil régional du Travail en temps de guerre pour le Québec qui surveillait l'application de cette politique, a permis à ceux qui lui en faisaient la demande, d'accorder à leurs salariés de tels congés. C'était une compensation que les employés ont recherchée avidernent puisque durant les années d'activité du Conseil, de lautomne 1941 à l'été 1946, il a accepté huit cent dix-huit requêtes affectant quatre cent quatorze mille cinq cent trente-deux salariés ${ }^{3}$. Les congés octroyés, dans le presque totalité des cas, étaient d'une semaine; les autres plans acceptés comportaient deux ou trois semaines de congés payés.

Dans l'ensemble, ces congés ont été subséquemment l'objet de clauses de conventions collectives de travail et de décrets en vertu de la Loi de la convention collective *, surtout lorsque cette Loi a été amendée de façon à permettre que les ${ }^{*}$ congés payés puissent être matière à juridiction ${ }^{5}$. Rappelons en effet que dans une étude sur le sujet ${ }^{6}$, dont un rapport succinct a paru dans cette revue ${ }^{7}$, sur cent conventions collectives conclues, dont quarante-sept ont donné naissance à un décret en vertu de la Loi de la convention collective, et cinquante-trois selon la Loi des syndicats professionnels au cours des années 1940 à 1945, quarante-quatre comportaient une clause de congés payés. Plus rẹ́cem-

(3) Statistiques obtenues du Conseil régional du travail en temps de guerre pour le Québec.

(4) S.R.Q., 1941 , c. 163 et amendements.

(5) 10 Geo. VI, c. 38 , a.1.

(6) R.P. Henri Légaré, Congés payés dans la province de Québec, mémoire présenté à la Faculté des sciences sociales de l'Université Laval, 1945.

(7) Bulletin des relations industrielles, vol. 2, numéro 9, mars 1946. 
ment, en 1947, soixante et un décrets sur quatrevingt-quatre alors en vigueur y pourvoyaient. C'était un progrès remarquable si l'on considère que dans le secteur de la législation quatre décrets seulement comportaient, en 1940, des dispositions à cet égard. Cette mesure sociale a cependant connu son essor le plus considérable lors de l'adoption par la Commission du salaire minimum de son ordonnance numéro $3^{8}$ qui venait accorder aux employés des établissements industriels et commerciaux un droit légal à des congés annuels payés.

Mais quel est donc le champ juridictionnel de cette réglementation et ses modalités d'application?

Nous croyons qu'il y a lieu au préalable de bien situer ce que l'on peut entendre par congés payés. L'ordonnance ne le spécifie pas, mais pour les fins de cette étude, nous pouvons citer la définition suivante suggérée à la Conférence internationale du Travail en 1935: "Par congé payé des salariés, on entend un nombre préalablement déterminé de journées consécutives autres que les jours fériés et les jours de maladie ou de convalescence, pendant lesquelles, chaque année, le salarié remplissant certaines conditions de service, interrompt son travail tout en continuant à recevoir sa rémunération habituelle. ${ }^{9}$

Si en principe une réglementation sur les congés annuels payés doit protéger tous les travailleurs, on conçoit facilement qu'en pratique et surtout à son origine, il soit difficile de les couvrir tous sans distinction. C'est probablement pourquoi l'ordonnance numéro 3 , dans sa juridiction, se limite à certains salariés des établissements industriels et commerciaux et élimine des groupes importants, comme les ouvriers forestiers ${ }^{10}$ dont le travail est exécuté en territoire non organisé. Notons aussi qu'elle ne couvre pas les salariés occupés à des exploitations agricoles et les domestiques de maison qui échappent à la juridiction de la Commission. De plus, les employés régis par les décrets en exécution de la Loi de la convention collective n'y sont pas soumis même si ces décrets ne contiennent aucune disposition particulière au sujet des congés payés. Ce sont des exceptions statutaires.

(8) Ordonnance no 3 du 14 septembre 1946, devenue l'ordonnance no 3 revisée le 8 février 1947.

(9) C.I.T. 19ième session, Genève 1935, Rapport V, Congés payés.

(10) Régis par l'ordonnance no 39, 1942, de la Commission du salaire minimum.
L'ordonnance est également inapplicable aux employés assujettis à une convention collective de travail (autre qu'un décret) comportant un plan de congés annuels payés d'une durée minimum d'une semaine.

Le droit au congé s'établit d'après la durée et la continuité des services chez le même employeur. Le minimum est d'une semaine après un an de service ininterrompu (une demi-journée par mois de calendrier de service continu). Cependant, l'année de base, pour les fins de calcul du congé, part du ler mai d'une année et se termine le 30 avril suivant. Cette année basique peut temporairement intervenir avec la coutume de certaines entreprises et devenir l'occasion de difficultés. Mais cette disposition, peut être tempérée par la signature d'une convention collective de travail comportant la règle d'un congé minimum payé d'une semaine. Cette exception a donc l'avantage de permettre aux employeurs qui négocient avec leurs salariés des conventions collectives, de prévoir des conditions plus compatibles avec leurs activités. Le régime idéal est encore celui que les intéressés peuvent eux-mêrnes établir à leur convenance et l'ordonnance l€ur laisse la voie libre. Aussi, les conventions collectives viennent en principe confirmer les dispositions réglementaires et établir, comme il arrive le plus souvent, un régime plus généreux.

Une ordonnance aussi générale puovait difficilement fixer des normes rigides quant à l'époque où ce congé devait être attribué. C'est pourquoi l'ordonnance accorde à l'employeur un délai d'un an après que l'employé a acquis son droit au congé. Il doit toutefois prévenir l'employé au moins quinze jours à l'avance de la date où il pourra en jouir.

La Commission, dans la détermination des règles de rétribution du congé, semble avoir voulu distinguer entre les salariés de service généralement engagés et payés à la semaine ou pour une période plus longue et dont le salaire est normalement régulier, et ceux de la production dont la rémunération se ramène à la base horaire et varie avec le rendement. Aussi trouve-t-on dans l'ordonnance deux modes de calcul qui tiennent compte des diverses modalités du salaire. En pratique, ces deux modes représentent deux pour cent du salaire gagné au cours de la période donnant droit au congé.

Autre fait d'intérêt à souligner, c'est que l'employé durant son congé a droit en plus de son salaire, à tous les avantages qui lui sont conventionnellement accordés, tels la pension ou son 
équivalent. Sauf dans les cas de résiliation de contrat de travail, l'ordonnance ne permet aucune compensation pour tenir lieu du congé et l'empioyé ne peut y renoncer. Bien plus, le droit acquis à un congé payé est inaliénable même s'il y a faute grave de salarié ou si encore, de son fait personnel, il met fin au contrat individuel de travail.

A l'exception de l'indemnité exigible lors de la résiliation de son contrat de travail, l'employé ne peut personnellement réclamer devant les tribunaux les congés et la rémunération dus. La Commission du salaire minimum, dans l'exercice de ses fonctions, doit voir à la bonne observance de l'ordonnance; elle a le pouvoir d'imposer à l'employeur en défaut une pénalité égale au double de la rémunération qu'il aurait dû payer au salarié pour son congé et dont la moitié peut être remise au salarié qui n’a pas obtenu de congé rémunéré.

Malgré ses imperfections apparentes, cette réglementation permet aux salariés, dans son ensemble, d'anticiper certains bienfaits physiques et moraux. Des améliorations notables y ont d'ail- leurs été apportées depuis son adoption et il $\mathrm{y}$ a lieu de croire que l'expérience acquise au cours de son application favorisera graduellement la précision de ses dispositions en même temps qu'elle visera à en faire bénéficier le plus grand nombre des travailleurs. Mais il reste qu'en principe les salariés qui n'en reçoivent pas encore ont toujours à leur disposition un merveilleux moyen d'atteindre cet idéal: la convention collective de travail.

Comment, en pratique, cette politique des congés payés servira-t-elle les salariés? Elle rendra les services prévus, à la condition seulement que tous les employeurs y collaborent sincèrement et qu'ensuite les employés utilisent les congés aux fins pour lesquelles ils sont devenus nécessaires. D’où l'on réalise déjà la nécessité impérieuse d'une organisation rationnelle des loisirs des travailleurs afin que les congés, normalement une source de bienfaits moraux et physiques, ne deviennent pas l'occasion de désordres sociaux. Les organisations professionnelles ont déjà fait beaucoup pour les travailleurs; leurs initiatives en maints domaines ont été couronnées de succès. Ce nouveau champ d'action ne pourrait-il pas maintenant faire l'objet de leur attention?

TABLEAU 1: DISTRIBUTION DES JOURS FÉRIÉS PAYES DANS UN GROUPE DE 560 CONVENTIONS COLlectives de tRAVAIL EN VIGUEUR DANS LA PROVINCE DE QUÉBEC, DECEMBRE 1947

\begin{tabular}{|c|c|c|c|c|c|c|c|c|c|c|c|c|c|c|c|c|}
\hline \multirow[t]{2}{*}{ Classe } & \multirow{2}{*}{$\begin{array}{l}\text { Total des } \\
\text { conventions } \\
\text { analysées }\end{array}$} & \multirow{2}{*}{$\begin{array}{l}\text { Total des con- } \\
\text { ventions com- } \\
\text { portant une } \\
\text { clause de jours } \\
\text { fériés payés }\end{array}$} & \multicolumn{14}{|c|}{$\begin{array}{c}\text { Nombre de conventions collectives comportant des } \\
\text { clauses de jours fériés payés d'après le nombre de } \\
\text { jours fériés payés }\end{array}$} \\
\hline & & & 1 & 2. & 3 & 4 & 5 & 6 & 7 & 8 & 9 & 10 & 11 & 12 & 13 & 14 \\
\hline Mines & 11 & 1 & 1 & & & & & & & & & & & & & \\
\hline $\begin{array}{l}\text { Industries manu- } \\
\text { facturières }\end{array}$ & 406 & 184 & 14 & 34 & 27 & 19 & 8 & 26 & 31 & 13 & 1 & 3 & 6 & 1 & 1 & \\
\hline Construction & 5 & 1 & & & & 1 & & & & & & & & & & \\
\hline $\begin{array}{l}\text { Transports et } \\
\text { commmunications }\end{array}$ & 15 & 5 & & 1 & & & & & & & & 2 & 1 & & & 1 \\
\hline Commerce & 25 & 12 & & & & 1 & & & & & 7 & 1 & 1 & 2 & & \\
\hline Finance & 18 & 11 & & & & & & 1 & 10 & - & & & & & & \\
\hline Services & 77 & 49 & & 3 & 2 & 3 & 2 & 5 & & 10 & 2 & & 4 & 2 & 1 & 15 \\
\hline Autres & 3 & 3 & & & & & & 1 & & 1 & & 1 & & & & \\
\hline Total & 560 & 266 & 15 & 38 & $29 \mid$ & 24 & 10 & $33 \mid$ & 41 & 24 & 10 & 7 & 12 & 5 & 2 & 16 \\
\hline
\end{tabular}


TABLEAU 2: DISTRIBUTION DES JOURS FÉRIÉS CHÔMÉS NON PAYÉS DANS UN GROUPE DE 560 CONVENTIONS COLLECTIVES DE TRAVAIL EN VIGUEUR DANS LA PROVINCE DE QUÉBEC, DÉCEMBRE 1947

\begin{tabular}{|c|c|c|c|c|c|c|c|c|c|c|c|c|c|c|c|}
\hline \multirow{3}{*}{ Classe } & \multirow{3}{*}{$\begin{array}{l}\text { Total des } \\
\text { conven- } \\
\text { tions ana- } \\
\text { lysées }\end{array}$} & \multicolumn{2}{|c|}{ Total des conventions } & \multirow{2}{*}{\multicolumn{12}{|c|}{$\begin{array}{l}\text { Nombre de conventions collectives de travail } \\
\text { comportant une clause de jours fériés chô- } \\
\text { més non payés d'après le nombre de jours } \\
\text { fériés chốmés non payés }\end{array}$}} \\
\hline & & \multirow{2}{*}{$\begin{array}{l}\text { comportant } \\
\text { une clause } \\
\text { de jours fé- } \\
\text { riés chômés } \\
\text { non payés* }\end{array}$} & \multirow{2}{*}{$\begin{array}{l}\text { ne compor- } \\
\text { tant aucune } \\
\text { clause de } \\
\text { jours fériés }\end{array}$} & & & & & & & & & & & & \\
\hline & & & & 1 & 2 & 3 & 4 & 5 & 6 & 7 & 8 & 9 & 10 & 11 & 12 \\
\hline Mines & 11 & 10 & & & & & & & & & 7 & 3 & & & \\
\hline $\begin{array}{l}\text { Industries manu- } \\
\text { facturières }\end{array}$ & 406 & 189 & 33 & & & 1 & 3 & 8 & 19 & 35 & 34 & 31 & 41 & 13 & 4 \\
\hline Construction & 5 & 3 & 1 & & & & & & 1 & & & 1 & 1 & & \\
\hline $\begin{array}{l}\text { Transports et } \\
\text { communications }\end{array}$ & 15 & 6 & 4 & & & & 1 & & 2 & 1 & 1 & 1 & & & \\
\hline Commerce & 25 & 10 & 3 & & & 1 & 2 & & & 1 & 3 & 2 & & & 1 \\
\hline Finance & 18 & 3 & 4 & & & & & & & 2 & 1 & & & & \\
\hline Services & 77 & 3 & 25 & & & & 1 & & & & 1 & & 1 & & \\
\hline Autres & 3 & & & & & & & & & & & & & & \\
\hline Total & 560 & 224 & 70 & & & 2 & 7 & 8 & 22 & 39 & 47 & 38 & 43 & 13 & 5 \\
\hline
\end{tabular}

\title{
UNE COMMISSION D'APPRENTISSAGE DES METIERS DU BÂTIMENT
}

\author{
J. L. E. PRICE
}

Pour satisfaire aux clauses de la Loi de l'aide à l'apprentissage la «Commission d'apprentissage des métiers du bâtiment et du génie de la construction de Montréal » fut incorporée en vertu d'un ordre-en-conseil, au moins de juin 1945. Incidemment, et pour plus de commodité dans l'usage courant, ce nom juridique a depuis été raccourci en celui de « Commission d'apprentissage des métiers du bâtiment de Montréal ».

Conformément aux termes de sa constitution officielle, la Commission, qui siège trimestriellement, est composée d'un nombre égal d'employeurs et d'employés: sur un total de 20 membres, 10 sont nommés par le Builders' Exchange de Montréal pour représenter les employeurs; les 10 autres représentent les travailleurs et sont nommés par des organisations ouvrières qui font partie du Cornité conjoint des métiers du bâtiment de Montréal.
Les agissements au jour le jour de la Commission sont contrôlés par un bureau de direction qui se réunit chaque semaine et se compose de huit membres de la Commission, dont quatre représentent les employeurs, et quatre, les employés.

Le président et le vice-président de la Commission font aussi office respectivement de président et de vice-président du bureau de direction.

Le centre de formation des métiers du bâtiment de Montréal, le premier du genre à s'établir dans la province, se loge à 2255 est, avenue Laurier, dans les locaux mis gratuitement à la disposition de la Commission par le gouvernement provincial.

Le personnel régulièrement à l'emploi du centre en février 1948 était composé d'un directeur, d'un assistant-directeur qui est en même temps un spécialiste en orientation professionnelle, un secrétaire-trésorier, un surintendant, six instructeurs- 